\title{
Up-dated the Critical Issues of Corneal Cross-linking (type-I and II): safety dose for ultra-thin cornea, demarcation line depth and the role of oxygen
}

\author{
Jui-Teng Lin ${ }^{*}$ \\ ${ }^{1}$ New Vision Inc. New Taipei City, Taiwan R.O.C. \\ *jtlin55@gmail.com
}

\begin{abstract}
Purpose: To update analytic formulas for the overall efficacy of corneal collagen crosslinking (CXL) including both type-I and oxygen-mediated type-II mechanisms, the role of oxygen and the initiator regeneration. Also to derive the formulas for the minimum corneal thickness and demarcation line depth.

Study Design: modeling the kinetics of CXL in UV light and using riboflavin as the photosensitizer. Place and Duration of Study: New Taipei City, Taiwan, between June, 2021 and July, 2021.

Methodology: Coupled kinetic equations are derived under the quasi-steady state condition for the 2-pathway mechanisms of CXL. For type-I CXL, the riboflavin (RF) triplet state [T] may interact directly with the stroma collagen substrate $[A]$ to form radical $(R)$ and regenerate initiator. For type-II process, $[T]$ interacts with oxygen to form a singlet oxygen $\left[{ }^{1} \mathrm{O}_{2}\right]$. Both reactive radical $(R)$ and $\left[{ }^{1} \mathrm{O}_{2}\right]$, can relax to their ground state, or interact with the substrate $[A]$ ) for crosslinking. Based on a safety dose, formulas for the minimum corneal thickness and demarcation line depth (DLD) are derived.

Results: Our updated theory/modeling showed that oxygen plays a limited and transient role in the process, in consistent with that of Kamave. In contrary, Kling et al believed that type-II is the predominant mechanism, which however conflicting with the epi-on CXL results. For both type-I and type-II, a transient state conversion (crosslink) efficacy in an increasing function of light intensity (or dose), whereas, its steady state efficacy is a deceasing function of light intensity. RF depletion in type-l is compensated by the RF regeneration term (RGE) which is a decreasing function of oxygen. For the case of perfect regeneration case (or when oxygen=0), RF is a constant due to the catalytic cycle. Unlike the conventional Dresden rule of 400 um thickness, thin cornea CXL is still safe as far as the dose is under a threshold dose $\left(E^{*}\right)$, based on our minimum thickness formula $\left(Z^{*}\right)$. Our formula for thin cornea is also clinically shown by Hafez et al for ultra thin $(214 \mathrm{~nm}) \mathrm{CXL}$.

Conclusion: For both type-I and type-II, a transient state conversion (crosslink) efficacy in an increasing function of light intensity (or dose), whereas, its steady state efficacy is a deceasing function of light intensity. CXL for ultra thin corneas are still safe, as far as it is under a threshold dose $\left(E^{\star}\right)$, based on our minimum thickness formula, which has a similar tend as that of demarcation line depth.
\end{abstract}

Keywords: Corneal crosslinking; efficacy; kinetic modeling; oxygen; riboflavin; ultraviolet light; safety dose; minimum thickness.

\section{INTRODUCTION}

Corneal collagen crosslinking $(C X L)$ have been one of the important clinical subjects in the past 15 years and the biomechanical properties of corneal tissue after CXL have been reported and summarized in a book [1]. The safety and efficacy issues of CXL have been reported theoretically [218]. The critical parameters influencing the efficacy of $C X L$ include: initial concentration and diffusion depth of riboflavin (RF) (for type-I CXL) and oxygen (for type-II CXL), quantum yield, UV light intensity, dose and irradiation duration. Most of the previous models [2-6] are not accurate due to the oversimplified assumptions of constant RF profiles, or non-depleted RF, or UV light intensity following the simple Beer-Lambert law (BLL). Standard (Dresden) protocols were revised for faster (accelerated) CXL based on Bunsen-Roscoe law (BRL) having a limited validation of UV maximum intensity [13]. Controversial efficacy issues of Dresden versus accelerated corneal crosslinking $(A-C X L)$ have been discussed recently by Lin [13] and a concentration-controlled method (CCM) to improve the efficacy of

*Corresponding author: E-mail: jtlin55@gmail.com; 
A-CXL was also proposed [14].

Schumacher et al [3] reported the non-oxygen-mediated (NOM) type-I CXL, in contrast to Kling et al [5] claiming that oxygen-mediated (OM) type-Il played the critical role of CXL efficacy. Furthermore, Kamaev et al [2] claimed that CXL is NOM-type-I dominant, while the OM-type-II only plays a limited and transient role. If Kling et al [5] were correct, then all the reported results of epi-on CXL and accelerated CXL would not be possible, since only minimum initial oxygen supply is available and the resupply (diffusion) of oxygen takes about 10 minutes [2]. The efficacy and similar kinetics were presented for anti-cancer photodynamic process $[15,16]$, which, however, have ignored the type-I mechanism.

Since the first human data of Wollensak et al in 2003 using the so-called Dresden protocol [1,19], the efficacy of accelerated and standard CXL were reported clinically for the roles of RF concentration and oxygen [20-29]. The depth-dependent efficacy and clinical outcomes for thin corneas were reported [30-33]. Recently Hafez et al reported the first CXL for ultra thin corneas [34].

This study will focus on more accurate analytic formulas than our previous model [17], based on a revised kinetic scheme. This article will also up-date the safety dose, minimum corneal thickness, and the role of oxygen and initiator regeneration, which provides a crosslink cycle for improved efficacy. The recent clinical results for ultra thin sub-400 um cases (with corneal thickness of 214 to 398 um) reported by Hafez et al [34] will be analyzed by the formulas of minimum corneal thickness and demarcation line depth (DLD).

\section{MATERIALS AND METHODS}

Both type-I and type-II reactions can occur simultaneously, and the ratio between these processes depends on the type of photosensitizers (PS) used, the concentrations of PS, substrate and oxygen, the kinetic rates involved in the process, and the light intensity, dose, PS depletion rate etc. [17]. Detailed kinetic of type-II only, and type-I only was published in my prior work [10] and [11], respectively. Typical depletion time of oxygen is about 5 to 15 seconds, for light intensity of 30 to $3 \mathrm{~mW} / \mathrm{cm}^{2}$, per measured data of Kamaev et al [2], and takes about 10 minutes for the oxygen to be resupplied or replenished to about $1 / 3$ of its initial state.

As shown in Figs. 1, the CXL process is described as follows. The ground state RF molecules (C) are excited by the UV light to its triplet excited state $(T)$. In type-I process, $(T)$ could interact directly with the stroma collagen substrate $[\mathrm{A}]$ for crosslinking, and produces a radical $(\mathrm{R})$ and regenerate the initiator (C). T could also interact with the ground state oxygen, $\left[\mathrm{O}_{2}\right]$, to form reactive superoxide anion radicals [O-] (not shown in Fig.1). For type-II process, T interacts with $\left[\mathrm{O}_{2}\right]$ to form oxygen singlet $\left[{ }^{1} \mathrm{O}_{2}\right]$, which could be relaxed to its ground state oxygen $\left[\mathrm{O}_{2}\right]$, or crosslink the stroma substrate $[\mathrm{A}]$. It could be used to kill bacteria for the treatment of corneal karatatitis or for anti-cancers.

The kinetic equations (based on the kinetic chart of Fig. 1) for the concentration of various components are shown as follows, by using short-hand notations: $\mathrm{C}$ and $\mathrm{T}$ for the RF ground and excited triplet state; $R$ for the active radical, $S$ for the singlet oxygen $\left[{ }^{1} \mathrm{O}_{2}\right]$; , and $X$ for the ground state oxygen $\left[{ }^{3} \mathrm{O}_{2}\right]$; and $[\mathrm{A}]$ for the available extracellular matrix substrate.

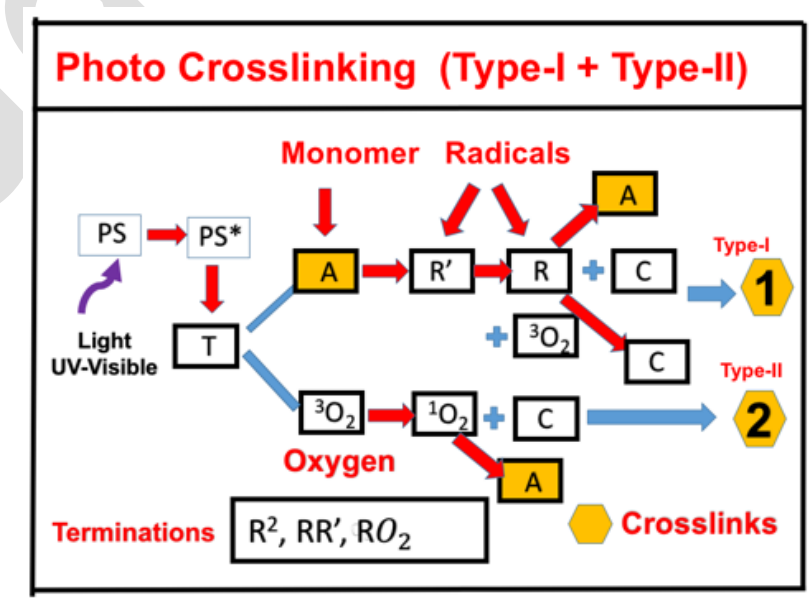

Fig. 2. The kinetics of CXL showing both type-I and -II pathways (see text for more details). 


$$
\begin{gathered}
\frac{d C}{d t}=-b I C+R G E \\
\frac{d T}{d t}=b I C-T / g \\
\frac{d R}{d t}=K_{3}[A] T-\left(k^{\prime} R+k^{\prime \prime} X+K_{1}[A]\right) R \\
\frac{d S}{d t}=k_{4} X T-\left(k_{6}+k_{1} C+K_{2}[A]\right) S \\
\frac{d X}{d t}=-\left(k^{\prime \prime} R+k_{4} T\right) X+k_{6} S+P \\
\frac{d[A]}{d t}=-\left(K_{3} T+K_{1} R+K_{2} S\right)[A] \\
R G E=1 / g+k^{\prime \prime} X R-k_{1} C S ~_{3}
\end{gathered}
$$

where, $g=1 /\left(k^{\prime \prime}+K_{3}[A]+k_{4} X\right)$ is the lifetime of the excited triplet state $(T) . \quad b=a$ 'q83.6 $\lambda$, with $q=k_{2} /\left(k_{1}+k_{2}\right)$ is the quantum yield of $T ; a^{\prime}$ is the extinction coefficients of RF ; $\lambda$ being the UV light wavelength. $g=1 /\left(k^{\prime \prime}+K_{3}[A]+k_{4} X\right)$ defining the lifetime of $T$ (given by $\left.1 / g\right)$; Eq. (1.e) includes an oxygen source term given by $P=\left(1-X / X_{0}\right) P_{0}$, with a maximum rate constant $P_{0}$, where $\left(1-X / X_{0}\right)$ is included to avoid the negative value of oxygen.

We note, in Eq. (1.a), that - bIC is the RF depletion, which is compensated by a regeneration term, $R G E$, such that $d C / d t=-(b I C-R G E)=-\left(k_{1} C S-k^{\prime} R X\right)$. However, the conventionally believed, that there is no RF depletion in type-II pathway is not totally correct, unless under a perfect cycle of RGE=bIC, which requires a perfect match of $k_{1} C S$ and $k " R X$. In fact, in a pure type-I case, with $X=S=0$, the perfect compensation (with RGE-bIC=0) is always valid, in the proposed scheme of Fig.1. For more complex schemes, this perfect cycle might not be met [18]. High efficacy requires a long lifetime of $R$ and $T$ (or large g). The conversion eq. (1.f) includes both terms for type-I ( $K_{3} T$ and $\left.K_{1} R\right)$ and type-II $\left(K_{2} S\right)$.

We note that Eq. (1) is much more complex and complete than that of Kamave et al [2], which is our special case when $C(t)$ is a constant (using a continuing resupply of $R F), k_{1} C=0$ in Eq. (1.d) and $k " R=0$ in Eq. (1.e). Our Eq.(1.f) has 3 terms for crosslink, but Kamave et al [2] ignored the $K_{1} R$, and assumed monomer conversion is only due to the coupling of $T$ and $[A]$, and the coupling of singlet oxygen (S) and [A]. Kinetic Equations of Schumacher et al [2] and Semchishen et al [4] are limited to the type-I conversion, $\mathrm{K}_{1} \mathrm{R}$, and ignored the oxygen-mediated term, $\mathrm{K}_{2} \mathrm{~S}$ in our Eq. (1.f). They also ignored the RGE cycle effects. The modeling of Kling [5] is based on Kamave [2], but only showed the algorithm for numerical calculations, there is no analytic formulas. Comparing to the above described previous modeling, our modeling, shown by Eq. (1), is the most complete and accurate one.

The dynamic UV light intensity is given by [11]

$$
\begin{gathered}
\frac{\partial \mathrm{I}(\mathrm{z}, \mathrm{t})}{\partial \mathrm{z}}=-\mathrm{A}^{\prime}(\mathrm{z}, \mathrm{t}) \mathrm{I}(\mathrm{z}, \mathrm{t}) \\
\mathrm{A}^{\prime}(\mathrm{z}, \mathrm{t})=2.3\left[\left(\mathrm{a}^{\prime}-\mathrm{b}^{\prime}\right) \mathrm{C}(\mathrm{z}, \mathrm{t})+\mathrm{b}^{\prime} \mathrm{C}_{0} \mathrm{~F}^{\prime}+\mathrm{Q}\right]
\end{gathered}
$$

where $F^{\prime}(z)=1-0.25 z / D$, with $D$ being the initial diffusion depth of $R F$; and $a=83.6 \lambda, \lambda$ being the UV light wavelength; $a^{\prime}=204(1 / \% / \mathrm{cm})$ and $b^{\prime}$ are the extinction coefficients of RF and the photolysis product, respectively; $Q=13.9(1 / \mathrm{cm})$ is the absorption coefficient of the stroma at the UV wavelength.

Comparing to our previous model $[10,17]$, we have revised the RGE term and proposed a revised pathway for type-I leading to radical $(R)$, via the coupling of $T$ and $[A]$, rather than $T$ and oxygen, whereas the type-II pathway remains the same. 
The kinetic equations (1) and (2) may be numerically calculated to find the CXL efficacy, which however is too complex for us to analyze the roles of each of the parameters. For comprehensive modeling we will use the so-called quasi-steady state assumption [15] described as follows. The life time of the triplet states of photosensitizer $(T)$ and the radical $(R)$ and singlet oxygen $(S)$ are very short (ns to $\mu$ s time scale) since they either decay or react with cellular matrix immediately after they are created. Thus, one may set, $d T / d t=d R / d t=d S / d t=0$, or the quasi-steady-state state. We obtain the steady-state solutions: $T=b l g C, S=g^{\prime} k_{4} T X$; with $g=1 /\left(k^{\prime \prime}+K_{3}[A]+k_{4} X\right) ; g^{\prime}=1 /\left(k_{6}+k_{1} C+K_{2}[A]\right)$. But radical $(R)$ is more complex given by the solution of

$$
k^{\prime} R^{2}+G R-H=0
$$

where $G=k " X+K_{1}[A]$ and $H=K_{3}[A] T$; with $T=b l g C$. Solving for $R$, we obtain

$$
\mathrm{R}=\left(\frac{1}{2 \mathrm{k}^{\prime}}\right)\left(-\mathrm{G}+\sqrt{\mathrm{G}^{2}+4 \mathrm{k}^{\prime} \mathrm{H}}\right)
$$

Analytic formulas of $\mathrm{R}$ is available under two special cases.

Case (i) for unimolecular termination dominant, or $G>>k^{\prime} H$, we obtain $R=K_{3}(b l g C[A] / G)(1-0.5 H / G)$, which is a linear increasing function of $H / G$, or blgC/G, for first-order with $0.5 \mathrm{H}<<\mathrm{G}$. In this case, there is a oxygen inhibition $(\mathrm{OIH})$ effect which reduces the radical $(R)$ and the efficacy, because $G$ is an increasing function of oxygen (or $X$ ), $G=G=k " X+K_{1}[A]$.

Case (ii) for bimolecular termination dominant, with $H \gg>G R$, we obtain, $R=\left[H / k^{\prime}\right]^{0.5}$. a nonlinear function of $\left[\mathrm{K}_{3}(\mathrm{blgC})[\mathrm{A}]\right]^{0.5}$, a square root function. In contrast to case (i), the OlH plays no role in case (ii).

\section{RESULTS AND DISCUSSION}

\subsection{Efficacy for Type-I and Type-II}

We note that Eq. (1.f) includes the type-I unimolecular process involving direct coupling of $T$ and the substrate $[A]$ producing radical $(R)$, whereas Eq. the type-II term is due to the singlet oxygen coupling with $[A]$. In the absence of oxygen (or when oxygen is depleted after the transient 5 to 20 seconds), $\mathrm{X}=\mathrm{S}=0$, until the resupply of external oxygen. Both type-I and type-II pathway can occur simultaneously, and the ratio between these processes depends on the type of photosensitizers (PS) used, the concentrations of PS, substrate and oxygen, the kinetic rates involved in the process, and the light intensity, dose, PS depletion rate etc. More details will be shown later.

According to the proposed mechanism of Kamaev et al [2], under aerobic conditions, they believe that CXL in the cornea is initiated mainly due to the direct interaction between the substrate and excited $\mathrm{RF}$ triplet $(\mathrm{T})$, whereas oxygen (and singlet oxygen) play a limited and transient role in the process. In contrary, Kling et al [3] believed that type-II is the predominant mechanism. Our new modeling system demonstrated theoretically that CXL using RF as the PS is predominated by the NOM term of type-I, or the direct coupling of triplet RF to the substrate [A], since the OM pathways (in both type-I and II) via singlet oxygen play a limited and transient role in the process per Kamaev et al [2], who proposed the mechanisms but did not develop the detailed macroscopic equations shown in this study.

\subsection{Analytic Formulas}

The solutions of the crosslink efficacy, given by Eq. (5) and (6) depend on the radicals $\mathrm{R}$ and $\mathrm{S}$, and the approximate form of the $g$ factors, $g=1 /\left(k^{\prime \prime}+K_{3}[A]+k_{4} X\right) ; g^{\prime}=1 /\left(k_{6}+k_{1} C+K_{2}[A]\right)$. We will focus on the case of $g=1 /\left(K_{3}[A]\right)$ and time-independent form of $g^{\prime}=1 /\left(k_{6}+k_{1} C_{0}+K_{2} A_{0}\right)$, , such that $T=b l C /\left(K_{3}[A]\right)$, $S=\left(k_{4} / k_{6}\right) T g ' X, R=\left(b I C / k^{\prime}\right)^{0.5}$. Using these approximated solutions and under the condition of $R G E=b I C$, such that $C=C_{0}$, is a constant, and such that $T^{\prime}=T^{\prime} /\left(K_{3}[A]\right), R=\left(T^{\prime} / k^{\prime}\right)^{0.5}$, with $T^{\prime}=b l C_{0}$.

Solving for Eq. (1.f) allows us to find the conversion (or crosslink) efficacy defined by $C E=1-[A] / A_{0}$, with $A_{0}$ being the initial concentration of the corneal substrate. For type-I, from Eq. (1.f), with $K_{2} S=0$, 


$$
\frac{\mathrm{d}[\mathrm{A}]}{\mathrm{dt}}=-\mathrm{T}^{\prime}-\mathrm{K}_{1} \sqrt{\left(\mathrm{T}^{\prime} / k^{\prime}\right)}[\mathrm{A}]
$$

Time integral of Eq. (5) gives first-order solution of [A], and we obtain

$$
C E=(1-F)-d^{\prime}(1+F)
$$

where $\mathrm{F}(\mathrm{t})=\exp (-\mathrm{dt}), \mathrm{d}=\mathrm{K}_{1}\left(\mathrm{bIC}_{0}\right)^{0.5}$ and $\mathrm{d}^{\prime}=\left(\mathrm{k}^{\prime} \mathrm{bIC}\right)^{0.5} /\left(\mathrm{X}_{0} \mathrm{~K}_{1}\right)=\mathrm{d} \mathrm{k}^{10.5} /\left(\mathrm{X}_{0} \mathrm{~K}_{1}{ }^{2}\right)$, which has a transient state $C E=d t-d^{\prime}(2-d t)=\left(d+2 d^{\prime}\right) t-2 d^{\prime}$; and steady state $C E=\left(1-d^{\prime}\right)$, a deceasing function of light intensity. This feature (under the constant $\mathrm{C}(\mathrm{t})=\mathrm{C}_{0}$ case, a perfect regeneration) is similar to our previous formulas based on $C(t)=C_{0} \exp (-b l g t)$, such that $F(t)$ becomes $F^{\prime}(t)=\exp [-d H(t)]$, with $H(t)=2\left[1-e x p\left(-0.5 d^{\prime \prime} t\right)\right] / d^{\prime \prime}$, with $d^{\prime \prime}=b l g$, which has a transient state, with $H(t)=t$, same as $F^{\prime}(t)=e x p(-d t)$. The steady state value $F^{\prime}=2 d / d "=2 K_{1}\left[C_{0} /(b l)\right]^{0.5}$, which has the similar feature and that of $F(t)$, but it is inverse proportional to $(\mathrm{bI})^{0.5}$, that is higher light intensity leads to lower conversion than that of lower light intensity. this feature will be shown alter in Fig.3, in comparing to type-II. We note that the OIH effect plays no role in this case (ii) of type-I process.

For type-II, we need to solve for oxygen, $X(t)$, from Eq. (1.e) first. For the case of $P=0$, we obtain the oxygen given by, for the first-order solution with $[A]=A_{0}$ in the function of $S=k_{4} g^{\prime} T X$, with $g^{\prime}=1 /\left(k_{6}+k_{1} C_{0}+K_{2} A_{0}\right)$,

$$
\begin{gathered}
\mathrm{X}(\mathrm{t})=\mathrm{X}_{0} \exp [-\mathrm{Dt}] \\
\mathrm{D}=\mathrm{k}_{4}\left(1-\mathrm{g}^{\prime}\right) \mathrm{T}^{\prime}+\mathrm{k}^{\prime \prime} \sqrt{\mathrm{T}^{\prime} / k^{\prime}}
\end{gathered}
$$

Time integral of Eq. (1.f), for $K_{1}=K_{3}=0$, only the $K_{2} S$ term, with $S=\left(k_{4} / K_{6}\right) g^{\prime} T X$, with $g^{\prime}=$ leads to

$$
\mathrm{CE}=1-\exp (-\mathrm{H})
$$

where $H(t)=p^{\prime}[1-\exp (-D t)] / D$, with $p^{\prime}=\left(k_{4} g^{\prime}\right)\left(b \mid A_{0} X_{0}\right)$, which has a transient state $C E=1-\exp \left(-p^{\prime} t\right)=p^{\prime} t$, but a steady state $C E=p^{\prime} / D$, which is a decreasing function of light intensity. Our Eq. (7.a) and (8) may be compared with the Eq. (5) and (11) of Kling et al [5], however their formulas are not expressive forms, including unknown parameter [EM] in the equations and can be solved only numerically. Their Fig. 6 showed the similar feature as our Eq. (8) that higher light intensity has a lower CE.

Eq. (6) and (8) are based on a constant $C(t)=C_{0}$ case (for a perfect regeneration, or in the absence of oxygen, $X=S=0)$. For more general case of $C(t)=C_{0} \exp (-b l g " t)$, with $g$ " is a time averaged value of $g^{\prime \prime}=\mid \mathrm{bg}-R G E=k_{1} C S+k^{\prime} R X$, due to oxygen; Eq. (7.a) is revised as $X(t)=X_{0} \exp \left[-\left(G+G^{\prime}\right)\right]$, with $G(t)=k_{4}\left(1-g^{\prime}\right) T\left[1-\exp \left(-d^{\prime \prime} t\right)\right] / d^{\prime \prime}$, and $G^{\prime}(t)=2 k^{\prime \prime}\left(T^{\prime} / k^{\prime}\right)^{0.5}\left[1-\exp \left(-0.5 d^{\prime \prime} t\right)\right] / d^{\prime \prime}$, with $d^{\prime \prime}=b l g G$. Time integral of Eq. (1.f) leads to a revised Eq. (8), a complex function needs numerical integration.

Above analytic formulas are more accurate than our earlier published results [17]. The numerical results of CE for type-I (with $P=0$ case) is shown in right figure of Fig. 2 [17], whereas, left figure shows results based on CE due to only the second term of Eq. (5) and for $\mathrm{C}_{0} \exp (-\mathrm{blgt}$ ).
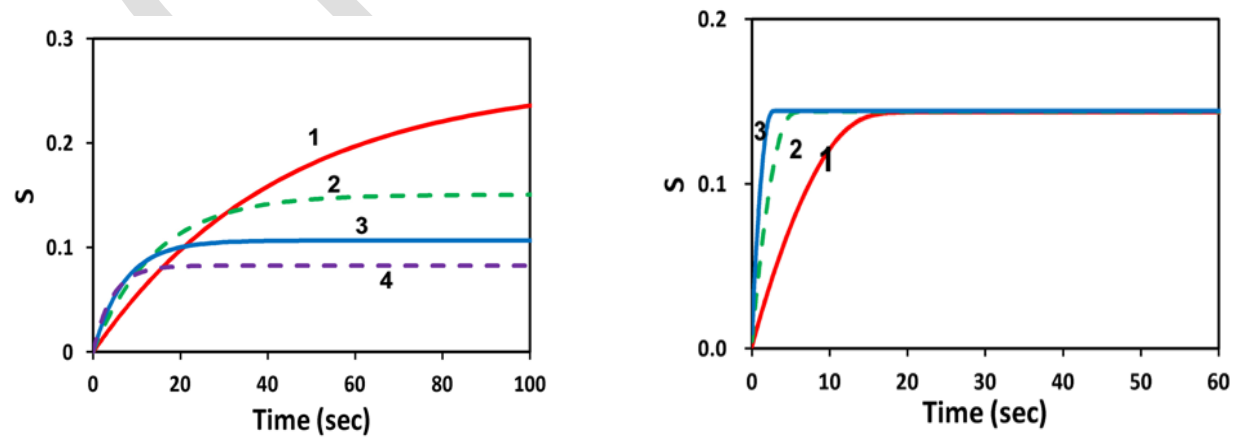

Fig. 2. The S-function profiles for Type-I (left) and type-II (right) , for intensity $I_{0}=(3,9,18,30)$ $\mathrm{mW} / \mathrm{cm}^{2}$ (curves $1,2,3,4$ ), for $\mathrm{C}_{0}=0.1 \%$, based on analytic formula Eq. (7) [17] 


\subsection{Minimum thickness and Demarcation line depth}

To estimate the safety dose and minimum thickness, we need to find the time (t) and depth ( $z$ ) dependence RF concentration, $C(t, z)$, and light intensity, I(z,t) given by the solution of Eq. (2) [ ] $I(z, t)=I_{0} \exp (-G)$, with $G$ is the integration of $A^{\prime}(z, t)$ over $z$, in which the concentration may be approximated by $C(z, t)=C_{0} \exp [-F(z, t)]$, with $F(z, t)$ is a time integral of $\left.\left(k_{1} S C-k^{\prime} R X\right)\right]$, which is a complex function of $z$ and $t$. However, for comprehensive formula, we could take a time and $z$ average of $F(z, t)=d ' t$, such that $C(z, t)=C_{0} \exp \left(-d^{\prime} t\right)$, which has a transient solution of $C_{0}\left(1-d^{\prime} t\right)$, and Eq. (2.b) becomes, for $F^{\prime}=1$ case, $A^{\prime}(z, t)=q^{\prime}-B t$, with $q^{\prime}=2.3\left(a^{\prime}+Q\right)$ and $B=2.3\left(a^{\prime}-b^{\prime}\right) d^{\prime}$, such that light intensity becomes $I(z, t)=I_{0}$ $\exp \left[-\left(q^{\prime}-B t\right) z\right]$, which is a revised time-dependent Beer Lambert law.

The light dose $(E)$ can be easily found by the time integral of $I(z, t)$ as $E(z, t)=I_{0} H(t) \exp \left(-q^{\prime} z\right)$, with $H(z, t)=[1-\exp (-B z t)] /(B z)$. Therefore, the corneal minimum thickness $\left(Z^{*}\right)$, defined by a damage dose threshold value of $E^{*}$ (or the safety dose). let $E=E^{*}$, and solve for $z=Z^{*}$, given by (for small $\left.B z t\right), E^{*}=E_{0}(1-$ $0.5 \mathrm{Bzt}) \exp \left(-\mathrm{q}^{\prime} z\right)$, which leads to the minimum corneal thickness given by

$$
Z^{*}=\left(1 / \mathrm{q}^{\prime}\right) \ln \left[\left(\mathrm{E}_{0} / E^{*}\right)\left(1-\mathrm{B} Z^{*} \mathrm{t}\right)\right]
$$

which has an analytic solution, when $B z t=0$. For small Bzt, $\ln (1-B z t)=-B z t$, Eq. (9) leads to $\left.Z^{*}=\left(1 / q^{\prime}\right) \ln (R) /[1+B t)\right]$, with $R=E_{0} / E^{*}$, which is time $(t)$ dependent due to the depletion of $C(t)$.

The demarcation line depth (DLD) may be defined by when as the efficacy is larger than a value ( $\left.E^{\prime}\right)$ for collagen tissue to be effectively affected and form the $D L D$. let $E_{0} \exp \left(-q^{\prime} Z^{\prime}\right)=E^{\prime}$, we obtain $D L D=Z^{\prime}=\left(1 / q^{\prime}\right) \ln \left(R^{\prime}\right)$, with ratio $R^{\prime}=E_{0} / E^{\prime}$ which has a similar trend as that of $Z^{*}$. The actual value of $Z^{\prime}$ may be calculated if the $E^{\prime}$ value can be measured accurately at a reference point.

\subsection{Analysis of measured data}

The recent clinical works of Hafez et al [33] showed safety cases for very thin corneas of (214 to $390 \mathrm{um}$ ), much less than the conventional minimum criterion of 400 um (based on the Dresden protocol). Our formula demonstrates the theoretical minimum thickness $\left(Z^{*}\right)$ could be as thin as 100 um (after epithelium removed), as far as the applied dose $\left(E_{0}\right)$ is less than the threshold value $\left(E^{*}\right)$. For example, for the case of $B z t=0$, Eq. (9) becomes $Z^{*}=\left(1 / q^{\prime}\right) \ln (R)$, with ratio $R=E_{0} / E^{*}$. For example, for $C_{0}=0.2 \%$, $a^{\prime}=204(1 / \% / \mathrm{cm})$, we obtain $q^{\prime}=2.3 a^{\prime} C_{0}=94(1 / \mathrm{cm})=0.0094(1 / \mathrm{um})$, approximated as $0.01(1 / \mathrm{um})$. Using $Z^{*}=100$ um for $R=2.72$, as the reference, then the safety thickness is given by $Z^{*}=(100 / 2.72) \ln \left(R^{\prime}\right)=(100$, $160,220,230,370,450)$ um, for $R=(2.72,4.5,8.0,12,20,33,55,90)$ and $\ln R=(1,1.5,2.0,2.5,3.0,3.5$, $4.0,4.5$ ), In The thin corneal thickness of Hafez et al [19] at $z=214$ um, corresponding to our $R=8.5$, or a dose of $E_{0}=8.5 E^{*}$ and $z=400$ um, for $R=55 E^{*}$. However, the actual value of $E^{*}$ and the referenced ratio $\left(R^{\prime}\right)$ require further clinical measurements. Hafez et al [33] reported the non-linear relation between the UV irradiation time and predicted demarcation line (referred to their Figure 3 and Table 2). They also showed the $Z^{*}$ vs. UV irradiation time (t), or dose for a fixed light intensity. However, the date (and curves) were based on the Dresden protocol of $t=30$ minutes, for $3 \mathrm{~mW} / \mathrm{cm}^{2}$ intensity, and an underestimated damage dose threshold $\left(E^{*}\right)$. Therefore, our formula based on the relative ratio of $E_{0} / E^{*}$ is much more accurate (if $E^{*}$ could be measured accurately).Figure 3 and Table 2 of Hafez et al[33] may be compared with our formula of $Z^{*}\left(1 / q^{\prime}\right) \ln \left(t_{0}\right)$, which is proportional to $\ln$ (irradiation time), for a fixed light intensity $\left(I_{0}\right)$. Similarly, The demarcation line depth (DLD) of their data follows our formulas.

\subsection{Summary of up-dated CXL Features}

From the analytic formulas Eq. (7) to Eq. (9) and the calculated data shown in Fig. 2, the key features of type-I and type-II CXL are summarized and compared as follows:

(a) Type-I and type-II coexit in CXL, in the presence of oxygen. However, there is no type-II when oxygen is depleted or in a condition without oxygen.

(b) Type-I has two cases: case (i) with unimolecular termination, the radical (R) and efficacy are a linear increasing function of blgC/G, but they are decreasing function of oxygen due to the $\mathrm{OlH}$ effect which reduces the radical $(R)$ and the efficacy, because $G$ is an 
increasing function of oxygen (or $X), G=G=k " X+K_{1}[A]$. In comparison, case (ii) for bimolecular termination, $R$ is a nonlinear square-root function of $\left[K_{3}(b \operatorname{lgC})[A]\right]^{0.5}$, but $\mathrm{OlH}$ plays no role.

(c) Oxygen is required for oxygen-mediated (OM) type-II and case(i) of type-I CXL, but it is not required in in case (ii) type-I. Therefore, type-II only plays a limited and transient state role for $t<t_{0}$, with to being the depletion time of oxygen.

(d) In the transient stage (about 3 to 20 seconds), both type-I and type-Il coexist until the oxygen is depleted; then type-I dominates before the oxygen is resupplied or replenished. The RF depletion is much slower than that of oxygen. Therefore, at the time oxygen is depleted, (or OM-type-II reaches its steady-state efficacy), approximately $60 \%$ to $80 \%$ of RF is still available to achieve NOM-type-I process.

(e) RF depletion in type-l is compensated by the RF regeneration term (RGE) which is a decreasing function of oxygen. For the case of perfect regeneration case (or $k_{1}[A]<<1 / g=0$ ), $R F$ is a constant due to the catalytic cycle.

(f) In type-II $C X L$, in the absence of oxygen supply (or $P_{0}=0$ ), higher intensity has a faster rising curve, but all intensities reach the same steady state value. However, for $P_{0}>0$, high intensity has lower steady state value due to the faster oxygen depletion-profiles.

(g) The overall CXL efficacy is governed by the time integration of $\mathrm{T}_{0}=\mathrm{bIC}$ (or $\mathrm{T}_{0}{ }^{0.5}$ ) for type-l; and bIC $\left[\mathrm{O}_{2}\right]$, for type-II. When either $\mathrm{C}$ or $\left[\mathrm{O}_{2}\right]$ is largely depleted, the $\mathrm{CXL}$ efficacy reaches its saturation level, which can not be improved by applying a higher dose (or longer exposure time), unless there are resupply of $C$ (via the $\mathrm{RGE}$ ) and/or $\left[\mathrm{O}_{2}\right]$ during the UV exposure. A so-called RF concentration-controlled method (CCM) was proposed for type-I [13]. Similarly, one may improve the type-II efficacy by external supply of high-pressure-oxygen, rather than its natural diffusion from air.

(h) The minimum corneal thickness $\left(Z^{*}\right)$ and the demarcation line depth (DLD, $\left.Z^{\prime}\right)$ are related to the similar formula given by, $Z^{*}=\left(1{ }^{\prime} q^{\prime}\right) \ln (R)$, and $Z^{\prime}=\left(1 / q^{\prime}\right) \ln \left(R^{\prime}\right)$, with $R=E_{0} / E^{*}$, and $E_{0} / E^{\prime}$, respectively.

The formulas developed in this study provide guidance for further clinical studies. The features predicted in this study are based on a modeling system which may not represent a real CXL system. Moreover, parameters (or the rate constants $\mathrm{k}_{\mathrm{j}}$ ) and thenthreshold dose $\left(\mathrm{E}^{*}\right)$ used in the calculatuons would require further clinical measurement for more accurate values. Greter details on the debating issues and a critical review on the kinetic and efficacy and optimal protocols of CXL will be published elsewhere.

\section{CONCLUSION}

Our new theory showed that oxygen (and singlet oxygen) play a limited and transient role in the process, in consistent with that of Kamave [2]. In contrary, Kling et al [3] believed that type-II is the predominant mechanism, which however conflicting with the epi-on CXL results. For both type-I and type-II, a transient state conversion (crosslink) efficacy in an increasing function of light intensity (or dose), whereas, its steady state efficacy is a deceasing function of light intensity. Ultra thin cornea is still safe as far as it is under a threshold dose $\left(E^{*}\right)$, based on our minimum thickness formula $\left(Z^{*}\right)$, as also clinically shown by Hafez et al [33]. However, the actual value of $E^{*}$ and the referenced ratio (R') require further clinical measurements.

\section{CONSENT}

It is not applicable.

\section{ETHICAL APPROVAL}

It is not applicable.

\section{COMPETING INTERESTS}


The author is the CEO of New Vision Inc. and has financial interest.

\section{REFERENCES}

1. Hafezi F and Randleman JB. editors. Corneal Collagen Cross-linking, second ed. Thorofare (NJ): SLACK; 2017.

2. Kamaev P, Friedman MD, Sherr E, Muller D. Cornea photochemical kinetics of corneal crosslinking with riboflavin. Vis. Sci. 2012;53:2360-2367.

3. Schumacher S, Mrochen M, Wernli J, Bueeler M, Seiler T. Optimization model for UV-riboflavin corneal cross-linking. Invest Opthamol Vis Sci. 2012; 53:762-769.

4. Semchishen A, Mrochen A, Semchishen V. Model for optimization of the UV-A/Riboflavin strengthening (cross-linking) of the cornea: percolation threshold. Photochemistry and photobiology, 2015; 91:1403-1411.

5. Kling S, Hafezi F. An algorithm to predict the biomechanical stiffening effect in corneal crosslinking. J Refract Surg 2017; 32:128-136. doi:10.3928/1081597X-20161206-01.

6. Hafezi F, Kling S, Gilardoni F, et al. Individualized corneal cross-linking with riboflavin and UV-A in ultra-thin corneas: the sub400 protocol. Am J Ophthalmol. 2021;224:133-142. doi:10.1016/j.ajo.2020.12.011

7. Lin JT, Liu HW, Cheng DC. On the dynamic of UV-light initiated corneal cross linking. J. Med Biolog Eng. 2014; 34: 247-250. doi: 10.5405/jmbe.15332.

8. Lin JT. Analytic formulas on factors determining the safety and efficacy in UV- light sensitized corneal cross-linking. Invest Ophthalmol Vis Sci 2015; 56:5740-574.

9. Lin JT. Combined analysis of safety and optimal efficacy in UV-light-activated corneal collagen crosslinking. Ophthalmology Research. 2016; 6(2):1-14. DOI\{10.9734/OR/2016/28712.

10. Lin JT. Photochemical Kinetic modeling for oxygen-enhanced UV-light-activated corneal collagen crosslinking. Ophthalmology Research, 2017;7:1-8. DOI: 10.9734/or/2017/35032.

11. Lin JT, Cheng DC. Modeling the efficacy profiles of UV-light activated corneal collagen crosslinking. PloS One. 2017;12:e0175002. DOI:10.1371/journal.pone.0175002.

12. Lin JT. Efficacy and $Z^{*}$ formula for minimum corneal thickness in UV-light crosslinking. Cornea, 2017: 36:30-31. DOI:10.1097/ico.0000000000001269.

13. Lin JT. A Critical Review on the Kinetics, Efficacy, Safety, Nonlinear Law and Optimal Protocols of Corneal Cross-linking. J Ophthalmology \& Visual Neuroscinece, 2018; 3:017.

14. Lin JT. A proposed concentration-controlled new protocol for optimal corneal crosslinking efficacy in the anterior stroma Invest. Ophthalmol Vis Sci. 2018 (in press).

15. Wernli J, Schumacher S, Spoerl E et al. The efficacy of corneal cross-linking shows a sudden decrease with very high intensity UV light and short treatment time. Invest Ophthalmol Vis Sci. 2013;54:1176-80. [PubMed].

16. Kim MM, Ghogare AA, Greer A, Zhu TC et al. On the in vivo photochemical rate parameters for PDT reactive oxygen species modeling. Phys. Med. Biol. 62 (2017) R1-R48.

17. Lin JT. Efficacy S-formula and kinetics of oxygen-mediated (type-II) and non-oxygen-mediated (type-I) corneal cross-linking. Ophthalmology Research. 2018; 8(1): 1-11.

18. Lin JT, Chen KT, Cheng DC, Liu HW. Modeling the efficacy of radical-mediated photopolymerization: the role of oxygen inhibition, viscosity and induction time. Front. Chem. 2019, 7:760. doi: 10.3389/fchem.2019.00760.

19. Wollensak G, Spoerl E, Wilsch M, Seiler T. Endothelial cell damage after riboflavin-ultraviolet-A treatment in the rabbit. J Cataract Refract Surg 2003;29:1786-90.

20. Lang PZ, Hafezi NL, Khandelwal SS, Torres-Netto EA, Hafezi F, Randleman JB. Comparative Functional Outcomes After Corneal Crosslinking Using Standard, Accelerated, and Accelerated With Higher Total Fluence Protocols. Cornea 2019;38:433-441.

21. Kanellopoulos AJ, Dupps WJ, Seven I, Asimellis G. Toric Topographically Customized Transepithelial, Pulsed, Very High-Fluence, Higher Energy and Higher Riboflavin Concentration Collagen Cross-Linking in Keratoconus. Case Report Ophthalmol 2014;5:172-180.

22. Seiler TG, Batista A, Frueh BE, Koenig K. Riboflavin Concentrations at the Endothelium During Corneal Cross-Linking in Humans. Invest Ophthalmol Vis Sci 2019;60:2140-2145.

23. Mazzotta C, Bagaglia SA, Vinciguerra R, Ferrise M, Vinciguerra P. Enhanced-fluence pulsed-light iontophoresis corneal cross-linking: 1-year morphological and clinical results. J Refract Surg 
2018;34:438-444

24. Mazzotta C, Bagaglia SA, Sgheri A et al.. Iontophoresis corneal cross-linking with enhanced fluence and pulsed UV-A light: 3-year clinical results. J Refract Surg 2020;36:286-292.

25. Mazzotta C, Sgheri A, Bagaglia SA, et al. Di Maggio A Customized corneal crosslinking for treatment of progressive keratoconus: Clinical and OCT outcomes using a transepithelial approach with supplemental oxygen. J Surg. 2020 Dec;46(12):1582-1587. doi: 10.1097/j.jcrs.0000000000000347.

26. Sachdev GS, Ramamurthy S, Dandapani R. Photorefractive intrastromal corneal crosslinking for treatment of low myopia: clinical outcomes using the transepithelial approach with supplemental oxygen. J Cataract Refract Surg 2020;46:428-433

27. Kling S, Richoz O, Hammer A, et al. Increased Biomechanical Efficacy of Corneal Cross-linking in Thin Corneas Due to Higher Oxygen Availability. J Refract Surg 2015;31:840-6.

28. Torres-Netto EA, Kling S, Hafezi N, Vinciguerra P, Randleman JB, Hafezi F. Oxygen Diffusion May Limit the Biomechanical Effectiveness of Iontophoresis-Assisted Transepithelial Corneal Crosslinking. J Refract Surg 2018;34:768-774.

29. Mazzotta C, Riomani A, Burroni A. Pachymetry-based Accelerated Cross-linking: The "M Nomogram" for Standardized Treatment of All-thickness Progressive Ectatic Corneas. Int K Kerat Ect Corn Dis 2019;7(2):137-144.

30. Jacob S, Kumar DA, Agarwal A, Basu S, Sinha P, Agarwal A. Contact Lens-Assisted Collagen Cross-Linking (CACXL): A New Technique for Cross-Linking Thin Corneas. J Refract Surg 2014;30:366-372.

31. Sachdev MS, Gupta D, Sachdev G, Sachdev R. Tailored stromal expansion with a refractive lenticule for crosslinking the ultrathin cornea. J Cataract Refract Surg 2015;41:918-23.

32. Zhang H, Roozbahani M, Piccinini AL, et al. Depth-Dependent Reduction of Biomechanical Efficacy of Contact Lens-Assisted Corneal Cross-linking Analyzed by Brillouin Microscopy. J Refract Surg 2019;35:721-728.

33. Hafezi F, Kling S, Gilardoni $F$ et al. Individualized corneal cross-linking with riboflavin and UV-A in ultra-thin corneas: the sub400 protocol. Am. J Ophthalmol. 2021;224:133-142. doi: https:// doi.org/10.1016/j.ajo.2020.12.011. 